\title{
A PARTIAL RESOLUTION OF THE PUNCTUAL HILBERT SCHEME OF A NONSINGULAR SURFACE
}

\author{
MARTIN G. GULBRANDSEN
}

\section{Introduction}

The punctual Hilbert scheme of a nonsingular surface is a variety whose closed points correspond to subschemes of finite length $n$, say, supported at a fixed point on the surface. It is singular in general. A less singular model has been suggested by A. S. Tikhomirov [8], namely a certain component of the variety parameterizing flags $\xi_{1} \subset \xi_{2} \subset \cdots \subset \xi_{n}$ of subschemes, where each $\xi_{i}$ has length $i$ and is supported at the chosen point. It is not obvious, however, how to determine whether a given flag belongs to this particular component. In this paper we show that a necessary, and at least for $n \leq 7$ sufficient, condition is that the associated filtration of ideals $I_{1} \supset I_{2} \supset \cdots \supset I_{n}$ has the multiplicative property $I_{i} I_{j} \subseteq I_{i+j}$. The variety parameterizing such flags can be algorithmically computed. In particular we find that the suggested model for the punctual Hilbert scheme is singular for $n=5$. This corrects an assertion of S. A. Tikhomirov's paper [9], where nonsingularity is erroneously claimed for $n=5$. In [8], A. S. Tikhomirov showed that the model is nonsingular for $n \leq 4$, a result we also obtain here.

In sections 2-4 we construct a scheme parameterizing flags of subschemes in a more general setting. In sections 5-6 we specialize to the case of a nonsingular surface.

I would like to thank Geir Ellingsrud for many valuable discussions. Also I thank Roy Skjelnes and the anonymous referee for useful comments.

\section{Punctual Hilbert schemes of flags}

Let $k$ be an algebraically closed field. By a scheme we shall mean a locally Noetherian scheme over $k$. Product of schemes means product over $k$ throughout. If $Y_{1}$ and $Y_{2}$ are closed subschemes of a third scheme $X$, the expression 
$Y_{1} \cap Y_{2}$ denotes their scheme theoretic intersection and $Y_{1} \subseteq Y_{2}$ means scheme theoretic inclusion. By a map of schemes we always mean a morphism in the category of schemes.

Let $(A, \mathfrak{m})$ be a local Artinian $k$-algebra of finite type. Then $X=\operatorname{Spec} A$ is a projective scheme, hence the Hilbert scheme $\operatorname{Hilb}^{n}(X)$ parameterizing subschemes $\xi \subset X$ of length $n$ exists [5].

Introduce the following notation: For a map of schemes $f: Y^{\prime} \rightarrow Y$, let

$$
f_{X}: Y^{\prime} \times X \longrightarrow Y \times X
$$

denote the product of $f$ with the identity map on $X$. Furthermore, for any scheme $Y$, let

$$
i_{Y}: Y \longrightarrow Y \times X
$$

denote the closed immersion obtained by identifying

$$
Y \cong Y \times \operatorname{Spec}(A / \mathfrak{m}) \subset Y \times X .
$$

To make formulas slightly more readable, we write $i_{*}^{Y}$ in place of $\left(i_{Y}\right)_{*}$ for push forward along $i_{Y}$.

We want to construct a scheme $\operatorname{Flag}^{n}(X)$ parameterizing complete flags of subschemes

$$
\xi_{1} \subset \cdots \subset \xi_{n} \subset X
$$

such that each $\xi_{i}$ has length $i$.

Definition 2.1. The Hilbert functor of complete flags in $X$ of length $n$ is the contravariant functor

$$
\text { Flag }^{n}(X): \mathrm{Sch}_{k} \longrightarrow \text { Sets }
$$

from the category of locally Noetherian schemes over $k$ to the category of sets that associates to a scheme $T$ the set of $n$-tuples of families

$$
T \times \operatorname{Spec}(A / \mathfrak{m})=W_{1} \subset \cdots \subset W_{n} \subset T \times X,
$$

with $W_{i}$ being defined by the ideal sheaf $\mathscr{J}_{i} \subset \mathscr{O}_{T \times X}$, such that

(I) each $W_{i}$ is flat and finite of degree $i$ over $T$

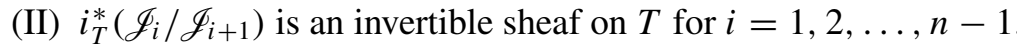

ReMARK 2.2. For $k$-valued points, condition (II) is automatic, thus a scheme representing $\operatorname{Flag}^{n}(X)$ does parameterize complete flags of subschemes in $X$. In fact, a $k$-valued point consists of subschemes $\xi_{i} \subset X$ of length $i$, defined by ideals

$$
I_{n} \subset \cdots \subset I_{1}=\mathfrak{m} \subset A .
$$


The sheaf $i_{T}^{*}\left(\mathscr{J}_{i} / \mathscr{J}_{i+1}\right)$ is now nothing but the $k$-vector space $I_{i} /\left(I_{i+1}+\mathfrak{m} I_{i}\right)$. Consider the obvious inclusions

$$
I_{i+1} \subseteq \mathfrak{m} I_{i}+I_{i+1} \subseteq I_{i}
$$

By Nakayama's lemma, the rightmost inclusion must be strict. By the assumption on lengths, the leftmost inclusion must then be an equality, that is, $\mathfrak{m} I_{i} \subseteq I_{i+1}$. Thus

$$
I_{i} /\left(I_{i+1}+\mathfrak{m} I_{i}\right)=I_{i} / I_{i+1}
$$

which is one-dimensional.

Similarly one can show that condition (II) is automatic for any reduced locally Noetherian base scheme $T$, but we shall not need this fact.

In the next section we shall prove the following result.

THEOREM 2.3. There exists a scheme $\operatorname{Flag}^{n}(X)$ representing $\operatorname{Flag}^{n}(X)$.

\section{Construction of $\operatorname{Flag}^{n}(X)$}

We construct Flag ${ }^{n}(X)$ by induction on $n$. For $n=1$ we clearly have $\operatorname{Flag}^{1}(X)=$ Spec $k$, with universal family

$$
Z_{1}=\operatorname{Spec} k \times \operatorname{Spec} k \subset \operatorname{Spec} k \times X \text {. }
$$

The main idea is the following: A closed point in $\operatorname{Flag}^{n}(X)$ corresponds to a filtration of ideals $I_{1} \supset \cdots \supset I_{n}$. Consider a closed point in $\mathrm{P}\left(I_{n} / \mathfrak{m} I_{n}\right)$, that is a vector space quotient

$$
I_{n} / \mathfrak{m} I_{n} \longrightarrow k \longrightarrow 0
$$

Such a quotient is also a homomorphism of $A$-modules, hence the kernel of the composite

$$
I_{n} \longrightarrow I_{n} / \mathfrak{m} I_{n} \longrightarrow k
$$

is an ideal $I_{n+1}$. The extended filtration $I_{1} \supset \cdots \supset I_{n} \supset I_{n+1}$ defines a closed point in $\operatorname{Flag}^{n+1}(X)$, and conversely any point arises in this way. The rest of this section is a straightforward globalization of this "fibrewise" construction.

Suppose now, for some fixed $n$, there exists a scheme $F=\operatorname{Flag}^{n}(X)$ representing $\operatorname{Flag}^{n}(X)$, and let

$$
Z_{1} \subset \cdots \subset Z_{n} \subset F \times X
$$

denote the universal flag, with $Z_{i}$ defined by the ideal sheaf $\mathscr{I}_{i} \subset \mathscr{O}_{F \times X}$. Define the coherent $\mathscr{O}_{F}$-module

$$
\mathscr{E}_{n}=i_{F}^{*} \mathscr{I}_{n}
$$


and let

$$
\pi: \mathrm{P}\left(\mathscr{E}_{n}\right) \longrightarrow F
$$

denote the structure map. We want to show that $\mathrm{P}\left(\mathscr{E}_{n}\right)$ represents $\operatorname{Flag}^{n+1}(X)$ by exhibiting a universal flag

$$
\widetilde{Z}_{1} \subset \cdots \subset \widetilde{Z}_{n+1} \subset \mathrm{P}\left(\mathscr{E}_{n}\right) \times X
$$

For $i=1, \ldots, n$, simply let

$$
\widetilde{Z}_{i}=\pi_{X}^{-1}\left(Z_{i}\right) \subset \mathrm{P}\left(\mathscr{E}_{n}\right) \times X
$$

which, since $Z_{i}$ is flat over $F$, is defined by the ideal sheaf

$$
\widetilde{I}_{i}=\pi_{X}^{*} \mathscr{I}_{i}
$$

Furthermore, we define

$$
\widetilde{Z}_{n+1} \subset \mathrm{P}\left(\mathscr{E}_{n}\right) \times X
$$

by the ideal sheaf $\widetilde{\mathscr{I}}_{n+1}$, constructed as follows: Let

$$
\phi_{1}: \widetilde{\mathscr{I}}_{n} \longrightarrow i_{*}^{\mathrm{P}\left(\mathscr{E}_{n}\right)} i_{\mathrm{P}\left(\mathscr{E}_{n}\right)}^{*} \widetilde{\mathscr{I}}_{n}=i_{*}^{\mathrm{P}\left(\mathscr{E}_{n}\right)} \pi^{* \mathscr{E}_{n}}
$$

be the canonical surjection and let

$$
\phi_{2}: i_{*}^{\mathrm{P}\left(\mathscr{E}_{n}\right)} \pi^{* \mathscr{E}_{n}} \longrightarrow i_{*}^{\mathrm{P}\left(\mathscr{E}_{n}\right)} \mathscr{O}(1)
$$

be the map obtained by applying $i_{*}^{\mathrm{P}\left(\mathscr{E}_{n}\right)}$ to the universal quotient

$$
\pi^{*} \mathscr{E}_{n} \longrightarrow \mathcal{O}(1) \longrightarrow 0
$$

on $\mathrm{P}\left(\mathscr{E}_{n}\right)$. Then define $\widetilde{\mathscr{I}}_{n+1}$ to be the kernel of $\phi_{2} \circ \phi_{1}$. The horizontal row in the following diagram is then exact:

$$
\begin{aligned}
& i_{*}^{\mathrm{P}\left(\mathscr{E}_{n}\right)} \pi^{* \mathscr{E}_{n}} \\
& \phi_{1} \nearrow \downarrow \\
& 0 \longrightarrow \tilde{\mathscr{I}}_{n+1} \longrightarrow \tilde{\mathscr{I}}_{n} \longrightarrow i_{*}^{\mathrm{P}\left(\mathscr{E}_{n}\right)} \mathcal{O}(1) \longrightarrow 0
\end{aligned}
$$

By the short exact sequence in (4) we see that $i_{\mathrm{P}\left(\mathscr{E}_{n}\right)}^{*}\left(\widetilde{\mathscr{I}}_{n} / \widetilde{\mathscr{I}}_{n+1}\right)$ is invertible, hence condition (II) in definition 2.1 is fulfilled. The same exact sequence may be rewritten

$$
0 \longrightarrow i_{*}^{\mathrm{P}\left(\mathscr{E}_{n}\right)} \mathcal{O}(1) \longrightarrow \mathcal{O}_{\widetilde{Z}_{n+1}} \longrightarrow \mathcal{O}_{\widetilde{Z}_{n}} \longrightarrow 0
$$


from which we see that $\widetilde{Z}_{n+1}$ is flat and finite of degree $n+1$ over $\mathrm{P}\left(\mathscr{E}_{n}\right)$, hence condition (I) is satisfied as well.

The following theorem ends the induction step and thus proves theorem 2.3:

THEOREM 3.1. The flag $\widetilde{Z}_{1} \subset \cdots \subset \widetilde{Z}_{n+1}$ constructed above has the following universal property: For any scheme $T$ and any $T$-valued point

$$
T \times \operatorname{Spec}(A / \mathfrak{m})=W_{1} \subset \cdots \subset W_{n+1} \subset T \times X
$$

of $\operatorname{Flag}^{n+1}(X)$, there exists a unique map

$$
f: T \longrightarrow \mathrm{P}\left(\mathscr{E}_{n}\right)
$$

such that $W_{i}=f^{-1}\left(\widetilde{Z}_{i}\right)$ for each $i$. Hence $\mathrm{P}\left(\mathscr{E}_{n}\right)$ represents $\underline{\text { Flag }}^{n+1}(X)$.

Proof. Let $\mathscr{J}_{i} \subset \mathcal{O}_{T \times X}$ be the sheaf of ideals defining $W_{i}$. By the induction hypothesis we have assumed that $F$ represents $\operatorname{Flag}^{n}(X)$, so the families $W_{1}, \ldots, W_{n}$ determine a unique map $g: T \rightarrow F$ such that $W_{i}=g_{X}^{-1}\left(Z_{i}\right)$ for $i=1, \ldots, n$. Since $Z_{i}$ is flat over $F$, the inverse image $g_{X}^{-1}\left(Z_{i}\right)$ is defined by $g_{X}^{*} \mathscr{I}_{i}$, hence $\mathscr{J}_{i}=g_{X}^{*} \mathscr{I}_{i}$. We want to show that $g$ extends uniquely to a map $f$ in the diagram

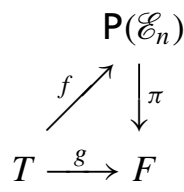

such that $f_{X}^{-1}\left(\widetilde{Z}_{n+1}\right)=W_{n+1}$, or equivalently $f_{X}^{*}\left(\widetilde{\mathscr{I}}_{n+1}\right)=\mathscr{J}_{n+1}$. Extending $g$ to a map $f$ in the diagram (5) is equivalent to giving a quotient

$$
g^{* \mathscr{E}} \mathscr{E}_{n} \longrightarrow \mathscr{L} \longrightarrow 0
$$

where $\mathscr{L}$ is an invertible sheaf on $T$. In fact, $f$ is then the unique map such that (6) is obtained by applying $f^{*}$ to the universal quotient (3).

Uniqueness: Assume there exists an $f$ in diagram (5) such that $f_{X}^{*}\left(\widetilde{\mathscr{I}}_{n+1}\right)=$ $\mathscr{J}_{n+1}$. We want to show that this determines the quotient (6) uniquely. This can be seen by applying $f^{*} i_{\mathrm{P}\left(\mathscr{E}_{n}\right)}^{*}$ to diagram (4). Firstly, applying $i_{\mathrm{P}\left(\mathscr{E}_{n}\right)}^{*}$ to the map $\phi_{1}$ in (1) we obtain the identity map on

$$
i_{\mathrm{P}\left(\mathscr{E}_{n}\right)}^{*} \widetilde{\mathscr{I}}_{n}=i_{\mathrm{P}\left(\mathscr{E}_{n}\right)}^{*} \pi_{X}^{*} \mathscr{I}_{n}=\pi^{*} i_{F}^{*} \mathscr{I}_{n}=\pi^{*} \mathscr{E}_{n}
$$

Furthermore, applying $i_{\mathrm{P}\left(\mathscr{E}_{n}\right)}^{*}$ to $\phi_{2}$ in (2) we recover the universal quotient (3). 
Thus, the result of applying $i_{\mathrm{P}\left(\mathscr{E}_{n}\right)}^{*}$ to diagram (4) is the following diagram:

$$
\begin{aligned}
& \|^{\pi^{* \mathscr{E}_{n}}} \\
& i_{\mathrm{P}\left(\mathscr{E}_{n}\right)}^{*} \tilde{\mathscr{I}}_{n+1} \longrightarrow i_{\mathrm{P}\left(\mathscr{E}_{n}\right)}^{*} \widetilde{\mathscr{I}}_{n} \longrightarrow \mathscr{O}(1) \longrightarrow 0
\end{aligned}
$$

Now applying $f^{*}$ and using the identity $i_{T}^{*} f_{X}^{*}=f^{*} i_{\mathbf{P}\left(\mathscr{E}_{n}\right)}^{*}$, we obtain

$$
i_{T}^{*} \mathscr{J}_{n+1} \longrightarrow i_{T}^{*} \mathscr{I}_{n} \stackrel{g^{* \mathscr{E}_{n}}}{\downarrow} \mathscr{L}^{\longrightarrow} \longrightarrow 0
$$

where $\mathscr{L}=f^{*} \mathscr{O}(1)$. Hence $f$ corresponds to the quotient

$$
i_{T}^{*} \mathscr{J}_{n} \longrightarrow i_{T}^{*}\left(\mathscr{J}_{n} / \mathscr{J}_{n+1}\right) \longrightarrow 0
$$

and is thus uniquely determined by the families $W_{i}$.

Existence: Simply define $\mathscr{L}=i_{T}^{*}\left(\mathscr{J}_{n} / \mathscr{J}_{n+1}\right)$ and let $f$ be the unique map corresponding to the quotient (8). This makes sense, since $\mathscr{L}$ is invertible by assumption. It remains only to check that we have $f_{X}^{*} \widetilde{\mathscr{J}}_{n+1}=\mathscr{J}_{n+1}$. For this, apply $f_{X}^{*}$ to the short exact sequence in (4) to obtain

$$
f_{X}^{*} \widetilde{\mathscr{I}}_{n+1} \longrightarrow \mathscr{J}_{n} \longrightarrow i_{*}^{T} \mathscr{L} \longrightarrow 0 .
$$

Now observe that the canonical map $\mathscr{J}_{n} / \mathscr{J}_{n+1} \rightarrow i_{*}^{T} \mathscr{L}$ is an isomorphism, under which the rightmost map in (9) may be identified with the canonical map $\mathscr{J}_{n} \rightarrow \mathscr{J}_{n} / \mathscr{J}_{n+1}$. Thus the kernel is $f_{X}^{*} \widetilde{\mathscr{I}}_{n+1}=\mathscr{J}_{n+1}$, that is, $f_{X}^{-1}\left(\widetilde{Z}_{n+1}\right)=$ $W_{n+1}$.

Proposition 3.2. The scheme $\operatorname{Flag}^{n}(X)$ is connected.

PRoof. If $f: X \rightarrow Y$ is a closed continuous surjective map of topological spaces, it is elementary that $X$ is connected if both $Y$ and the fibers of $f$ are. We apply this to the structure map

$$
\mathrm{P}\left(\mathscr{E}_{n}\right) \longrightarrow \operatorname{Flag}^{n}(X) .
$$

This map is proper and the fibers are projective spaces. Hence $\operatorname{Flag}^{n+1}(X)=$ $\mathrm{P}\left(\mathscr{E}_{n}\right)$ is connected if $\operatorname{Flag}^{n}(X)$ is. The conclusion follows by induction on $n$. 


\section{Punctual Hilbert schemes of multiplicative flags}

Definition 4.1. A $k$-valued point in $\operatorname{Flag}^{n}(X)$, corresponding to a filtration of ideals

$$
I_{n} \subset \cdots \subset I_{1}=\mathfrak{m} \subset A
$$

is multiplicative if we have $I_{i} I_{j} \subseteq I_{i+j}$ for all $i+j \leq n$.

We next construct a subscheme of $\operatorname{Flag}^{n}(X)$, parameterizing only multiplicative flags in $X$.

DEFINITION 4.2. The Hilbert functor of multiplicative complete flags in $X$ of length $n$ is the contravariant functor

$$
\underline{\operatorname{Mult}}^{n}(X): \operatorname{Sch}_{k} \longrightarrow \text { Sets }
$$

from the category of locally Noetherian schemes over $k$ to the category of sets that associates to a scheme $T$ the set of $n$-tuples of families

$$
T \times \operatorname{Spec}(A / \mathfrak{m})=W_{1} \subset \cdots \subset W_{n} \subset T \times X,
$$

with $W_{i}$ being defined by the ideal sheaf $\mathscr{J}_{i} \subset \mathscr{O}_{T \times X}$, such that

(I) each $W_{i}$ is flat and finite of degree $i$ over $T$

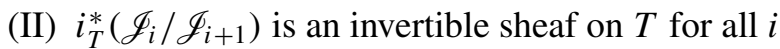

(III) $\mathscr{J}_{i} \mathscr{J}_{j} \subseteq \mathscr{J}_{i+j}$ for all $i+j \leq n$.

We want to show that the condition $\mathscr{J}_{i} \mathscr{F}_{j} \subseteq \mathscr{J}_{i+j}$ is closed, in the strong sense that $\operatorname{Mult}^{n}(X)$ is a closed subfunctor of $\operatorname{Flag}^{n}(X)$. This is a consequence of the following lemma:

Lemma 4.3. Let $\pi: Y \rightarrow S$ be a morphism of locally Noetherian schemes and let $W, Z \subseteq Y$ be closed subschemes such that $Z$ is flat and finite over $S$. Then there exists a unique $S$-scheme

$$
i: S^{\prime} \longrightarrow S
$$

such that

(I) $Z \times{ }_{S} S^{\prime} \subseteq W \times{ }_{S} S^{\prime}$

(II) if $T \rightarrow S$ is any $S$-scheme satisfying $Z \times{ }_{S} T \subseteq W \times{ }_{S} T$ then there exists a unique morphism $g: T \rightarrow S^{\prime}$ over $S$.

Furthermore, $i$ is a closed immersion.

Proof. Suppose the lemma holds whenever $S$ is affine. Then we may apply the lemma to each $S_{\alpha}$ in an affine open cover $\left\{S_{\alpha}\right\}$ of $S$. Thus there exists closed 
immersions $i_{\alpha}: S_{\alpha}^{\prime} \rightarrow S_{\alpha}$, uniquely determined by properties (I) and (II) when replacing $S, W$ and $Z$ with $S_{\alpha}, W \cap S_{\alpha}$ and $Z \cap S_{\alpha}$. Again applying the lemma to an affine open cover of each intersection $S_{\alpha} \cap S_{\beta}$, we see that the immersions $\left\{i_{\alpha}\right\}$ agree on the overlaps. Hence they may be glued to form the required closed immersion $i: S^{\prime} \rightarrow S$. Thus we may assume $S$ is affine.

Since $Z$ is finite over $S, Z$ is affine as well. Then we may choose a free presentation

$$
\mathcal{O}_{Z}^{n} \stackrel{\phi}{\longrightarrow} \mathcal{O}_{Z} \longrightarrow \mathcal{O}_{Z \cap W} \longrightarrow 0
$$

where $Z \cap W$ denotes the scheme theoretic intersection. Let $f: T \rightarrow S$ be any morphism, and let $\widetilde{Z}=Z \times{ }_{S} T$ and $\widetilde{W}=W \times{ }_{S} T$. We claim the condition $\widetilde{Z} \subseteq \widetilde{W}$ is equivalent to requiring $f^{*} \pi_{*} \phi=0$ : Form the fibre square

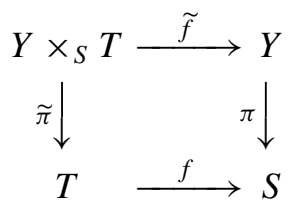

Then applying $\tilde{f}^{*}$ to (10) gives a free presentation of the structure sheaf of $\widetilde{Z} \cap \widetilde{W}$ :

$$
\mathscr{O}_{\widetilde{Z}}^{n} \stackrel{\tilde{f}^{*} \phi}{\longrightarrow} \mathcal{O}_{\widetilde{Z}} \longrightarrow \mathcal{O}_{\widetilde{Z} \cap \tilde{W}} \longrightarrow 0
$$

Thus the condition $\widetilde{Z} \subseteq \widetilde{W}$, or equivalently $\widetilde{Z} \cap \widetilde{W}=\widetilde{Z}$, is the same thing as requiring $\widetilde{f}^{*} \phi=0$. Now the restriction of $\tilde{\pi}$ to $\widetilde{Z}$ is finite, hence affine, so $\tilde{f}^{*} \phi=0$ if and only if $\tilde{\pi}_{*} \tilde{f}^{*} \phi=0$. Furthermore, as $Z$ is flat over $S$, $\widetilde{\pi}_{*} \widetilde{f}^{*} \phi=f^{*} \pi_{*} \phi$. Hence $\widetilde{Z} \subseteq \widetilde{W}$ if and only if $f^{*} \pi_{*} \phi=0$ as claimed.

Since $Z$ is flat and finite over $S$,

$$
\pi_{*} \mathcal{O}_{Z}^{n} \stackrel{\pi_{*} \phi}{\longrightarrow} \pi_{*} \mathcal{O}_{Z}
$$

is a map of locally free sheaves of finite rank on $S$. Thus $\pi_{*} \phi$ can be locally represented by a matrix of regular functions, hence its vanishing locus has a canonical structure of a closed subscheme $i: S^{\prime} \rightarrow S$. Then $i^{*} \pi_{*} \phi=0$, so $i$ has property (I). Furthermore, if a morphism $f: T \rightarrow S$ satisfies $f^{*} \pi_{*} \phi=0$, then the image in $\mathscr{O}_{T}$ of the ideal sheaf defining $S^{\prime} \subset S$ is zero, which says that $f$ factors through $i$. So $i$ has property (II).

THEOREM 4.4. $\operatorname{Mult}^{n}(X)$ is a closed subfunctor of $\operatorname{Flag}^{n}(X)$.

Proof. Let $S$ denote a scheme and $h_{S}$ its functor of points. Consider a 
cartesian diagram

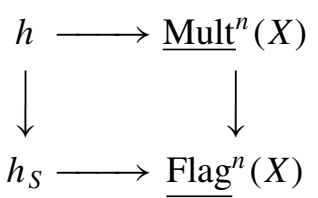

where $h$ is the fibre product functor. We claim there exists a closed subscheme $S^{\prime} \subseteq S$ and an isomorphism $h \cong h_{S^{\prime}}$ such that the map $h \rightarrow h_{S}$ is compatible with the inclusion map $h_{S^{\prime}} \rightarrow h_{S}$.

The image of a morphism $T \rightarrow S$ under the given map $h_{S} \rightarrow{\underline{\operatorname{Flag}^{n}}}^{n}(X)$ is a flag

$$
W_{1} \subset \cdots \subset W_{n} \subset X \times T .
$$

Let $\mathscr{J}_{i} \subset \mathcal{O}_{X \times T}$ denote the ideal sheaf corresponding to $W_{i}$. By definition, $h$ is the subfunctor of $h_{S}$ whose $T$-valued points are the morphisms $T \rightarrow S$ such that the corresponding flag (12) has the multiplicative property

$$
\mathscr{J}_{i} \mathscr{J}_{j} \subseteq \mathscr{J}_{i+j} \quad \text { for all } \quad i+j \leq n .
$$

Thus our claim is that there is a closed subscheme $S^{\prime} \subseteq S$ such that $T \rightarrow S$ factors through $S^{\prime}$ if and only if property (13) holds. This can be seen as follows:

The image of the identity map $\operatorname{id}_{S}$ under the given map $h_{S} \rightarrow \operatorname{Flag}^{n}(X)$ is a flag

$$
Z_{1} \subset \cdots \subset Z_{n} \subset X \times S
$$

over $S$, with $Z_{i}$ corresponding to some ideal sheaf $\mathscr{I}_{i} \subset \mathscr{O}_{X \times S}$. For any morphism $T \rightarrow S$, the corresponding flag (12) is just the pullback of the flag (14) along $T \rightarrow S$. Thus the existence of $S^{\prime} \subseteq S$ is a consequence of lemma 4.3, applied to $Y=X \times S, W=V\left(\mathscr{I}_{i} \mathscr{I}_{j}\right)$ and $Z=Z_{i+j}$, for each $i$ and $j$.

Corollary 4.5. There exists a closed subscheme $\operatorname{Mult}^{n}(X) \subseteq \operatorname{Flag}^{n}(X)$ representing Mult $^{n}(X)$.

Remark 4.6. The scheme $\operatorname{Mult}^{n}(X)$ can be constructed more explicitly in the same fashion that we constructed $\operatorname{Flag}^{n}(X)$ : Consider the universal flag

$$
Z_{1} \subset \cdots \subset Z_{n} \subset \operatorname{Flag}^{n}(X) \times X,
$$

with $Z_{i}$ defined by the ideal sheaf $\mathscr{I}_{i}$. Denote by

$$
W_{1} \subset \cdots \subset W_{n} \subset \operatorname{Mult}^{n}(X) \times X
$$

their restriction to $\operatorname{Mult}^{n}(X)$, with $W_{i}$ defined by the ideal sheaf $\mathscr{L}_{i}$. In section 3 we constructed $\operatorname{Flag}^{n+1}(X)$ as $\mathrm{P}\left(\mathscr{E}_{n}\right)$, where $\mathscr{E}_{n}=i_{F}^{*} \mathscr{I}_{n}$. Thus $\operatorname{Mult}^{n+1}(X)$ is 
the maximal subscheme of $\mathrm{P}\left(\mathscr{E}_{n}\right)$ such that the restriction of the universal flag has the multiplicative property. This is precisely the universal property of

$$
\pi: \mathrm{P}\left(\mathscr{F}_{n}\right) \longrightarrow \operatorname{Mult}^{n}(X)
$$

where

$$
\mathscr{F}_{n}=\mathscr{J}_{n} / \sum_{i=0}^{n-1} \mathscr{J}_{i+1} \mathscr{J}_{n-i},
$$

considered as a coherent sheaf on $\operatorname{Mult}^{n}(X) \cong W_{1} \subset \operatorname{Mult}^{n}(X) \times X$. Thus we have an isomorphism $\operatorname{Mult}^{n+1}(X) \cong \mathrm{P}\left(\mathscr{F}_{n}\right)$ over $\operatorname{Mult}^{n}(X)$. The universal multiplicative flag

$$
\widetilde{W}_{1} \subset \cdots \subset \widetilde{W}_{n+1} \subset \operatorname{Mult}^{n+1}(X) \times X
$$

is defined by ideals $\widetilde{\mathscr{J}}_{1} \supset \cdots \supset \widetilde{\mathscr{J}}_{n+1}$ where $\widetilde{\mathscr{J}}_{i}=\pi_{X}^{*} \mathscr{\mathscr { J }}_{i}$ for $i \leq n$, whereas $\widetilde{\mathscr{J}}_{n+1}$ is the kernel of the canonical map

$$
\widetilde{\mathscr{J}}_{n} \longrightarrow i_{*}^{\mathrm{P}\left(\mathscr{F}_{n}\right)} \mathscr{O}(1)
$$

where $\mathscr{O}(1)$ now denotes the tautological invertible sheaf on $\mathrm{P}\left(\mathscr{F}_{n}\right)$.

Proposition 4.7. The scheme $\operatorname{Mult}^{n}(X)$ is connected.

Proof. Using the construction of Mult ${ }^{n}(X)$ in remark 4.6, the proof of 3.2 can be repeated.

\section{Punctual Hilbert schemes of points on a nonsingular surface}

For the rest of this text we consider the following situation: Assume $k$ has characteristic zero. Fix an algebraic surface $S$ over $k$ and a nonsingular point $p \in S$. Let $\mathscr{O}_{S, p}$ denote the local ring at $p$ and let $\mathfrak{m}_{p} \subset \mathscr{O}_{S, p}$ denote its maximal ideal. Any subscheme $\xi \subset S$ of length $n$ and supported at $p$ is contained in the $(n-1)$ 'st infinitesimal neighbourhood $X=\operatorname{Spec} \mathscr{O}_{S, p} / \mathfrak{m}_{p}^{n}$. Thus the scheme $\operatorname{Hilb}^{n}(X)$ parameterizes length $n$ subschemes of $S$ supported at $p$. We let

$$
H(n)=\operatorname{Hilb}^{n}(X)_{\text {red }}
$$

denote the underlying reduced subscheme. We suppress $S$ and $p$ from the notation, as the definition of $H(n)$ only depends on the $(n-1)$ 'st infinitesimal neighbourhood of $p$, whose isomorphism class is independent of the choices of $S$ and $p$.

It is well known that $H(n)$ is irreducible and has dimension $n-1$ (proved by Briançon [1] over the complex numbers, see e.g. Ellingsrud and Lehn [2] for a proof in a more general setting). However, it is singular in general. For instance, $H(3)$ is isomorphic to the projective cone over the twisted cubic in 
$\mathrm{P}^{3}$. In the rest of this paper we present work towards finding a natural resolution of singularities of $H(n)$.

Following Le Barz [7], we make the following definition:

Definition 5.1. A subscheme $\xi \subset S$, supported at $p$, is curvilinear if there exists a curve $C$ which contains $\xi$ and is nonsingular at $p$.

It is well known ([1], [6]) that the subset of $H(n)$ consisting of curvilinear subschemes is open, dense and nonsingular. The following result is also well known:

Lemma 5.2. Let $\xi \subset S$ be a subscheme supported at a point $p$. If $\xi$ is curvilinear, there is a unique flag

$$
\xi_{1} \subset \cdots \subset \xi_{n-1} \subset \xi
$$

with $\xi_{i}$ of length $i$. In fact, $\xi_{i}$ is the intersection of $\xi$ with the $(i-1)$ 'st infinitesimal neighbourhood of $p$ in $S$.

Proof. Suppose $C$ is a nonsingular curve through $p$ containing $\xi$, locally defined by the ideal $J \subset \mathscr{O}_{X, p}$. Let $\xi_{i} \subset \xi$ be a subscheme of length $i$ and let $I \subset I_{i} \subset \mathscr{O}_{X, p}$ be the ideals defining $\xi$ and $\xi_{i}$. Then we have $\mathfrak{m}_{p}^{i} \subseteq I_{i}$, hence

$$
J+\mathfrak{m}_{p}^{i} \subseteq I+\mathfrak{m}_{p}^{i} \subseteq I_{i} .
$$

But the left hand side is the ideal defining the $(i-1)$ 'st infinitesimal neighbourhood of $p$ in $C$, which has colength $i$ since $C$ is nonsingular. Since the right hand side ideal $I_{i}$ has colength $i$ also, the inclusions are actually equalities. In particular $I_{i}=I+\mathfrak{m}_{p}^{i}$, which shows that $\xi_{i}$ is uniquely determined as the intersection of $\xi$ with the $(i-1)$ 'st infinitesimal neighbourhood of $p$ in $S$.

Define

$$
H F(n)=\operatorname{Flag}^{n}(X)_{\text {red }}
$$

which is a reduced scheme whose closed points correspond to flags of subschemes in $S$ supported at $p$. The canonical map

$$
\operatorname{Flag}^{n}(X) \longrightarrow \operatorname{Hilb}^{n}(X)
$$

induces a map

$$
\rho_{n}: H F(n) \longrightarrow H(n) .
$$

Proposition 5.3. There is a unique component $H F^{\prime}(n) \subseteq H F(n)$ which is mapped birationally onto $H(n)$ by $\rho_{n}$.

Proof. Let $U \subseteq H(n)$ be the open subset corresponding to curvilinear subschemes. By lemma 5.2, the fibre $\rho_{n}^{-1}(\xi)$ is a single point for every (closed) 
point $\xi \in U$. Hence $\rho_{n}$ is bijective over $U$. Since $\rho_{n}$ is proper and $U$ is nonsingular, Zariski's main theorem [4, prop. 4.4.1] shows that $\rho_{n}$ is an isomorphism over $U$. Thus the closure $H F^{\prime}(n)$ of $\rho_{n}^{-1}(U)$ in $H F(n)$ is the unique component mapping birationally onto $H(n)$.

Denote by

$$
\rho_{n}^{\prime}: H F^{\prime}(n) \longrightarrow H(n)
$$

the restricted map. We call this a partial resolution of $H(n)$. This construction has been studied by Tikhomirov in [8], where he proves that $\rho_{n}^{\prime}$ is a resolution of singularities for $n \leq 4$. The problem addressed in the next section is how to determine whether a given flag belongs to the component $H F^{\prime}(n)$. This leads us to a different proof of Tikhomirov's result (theorem 6.1) and also the new result that $H F^{\prime}(5)$ is singular (theorem 6.2).

Define

$$
\operatorname{HMF}(n)=\operatorname{Mult}^{n}(X)_{\text {red }}
$$

which is a reduced scheme whose closed points correspond to multiplicative flags of subschemes in $S$ supported at $p$. Since $\operatorname{Mult}^{n}(X)$ is a closed subscheme of $\operatorname{Flag}^{n}(X)$, we find that $H M F(n)$ is a closed subscheme of $H F(n)$. The motivation for studying $\operatorname{HMF}(n)$ is the following observation:

Proposition 5.4. Any (closed) point in $H F^{\prime}(n)$ is multiplicative, hence $H F^{\prime}(n)$ is contained in $H M F(n)$.

Proof. Denote by $U \subseteq H(n)$ the open set consisting of curvilinear points. Let $V \subseteq H F^{\prime}(n)$ denote the inverse image of $U$ by the map $\rho_{n}^{\prime}: H F^{\prime}(n) \rightarrow$ $H(n)$. By definition, $H F^{\prime}(n)$ is the closure of $V$ in $H F(n)$.

First consider a (closed) point in $V$, that is, a flag

$$
\xi_{1} \subset \cdots \subset \xi_{n}
$$

with $\xi_{n}$ curvilinear. Then, if $\xi_{i}$ corresponds to the ideal $I_{i} \subset \mathcal{O}_{X, p}$ we have

$$
I_{i}=\mathfrak{m}_{p}^{i}+I_{n} \quad \text { for all } \quad i
$$

by lemma 5.2. Then it is obvious that $I_{i} I_{j} \subseteq I_{i+j}$.

Thus $V \subset H M F(n)$. Since $H M F(n)$ is closed in $H F(n)$ and $H F^{\prime}(n)$ is the closure of $V$, we have $H F^{\prime}(n) \subset H M F(n)$.

QUESTION 5.5. Is the converse to proposition 5.4 true, i.e. do we have an equality $H F^{\prime}(n)=H M F(n)$ ? As $H F^{\prime}(n)$ is a component of $H F(n)$, this is equivalent to asking whether $\operatorname{HMF}(n)$ is irreducible. 
The calculations in section 6 show that the answer to the question is positive for $n \leq 7$. For higher $n$ we do not know. We remark that $\operatorname{HMF}(n)$ is at least connected, by proposition 4.7 .

\section{Examples}

To describe $H M F(n)$, we follow the construction of $\operatorname{Mult}^{n}(X)$ in remark 4.6. More explicitly, let $U=\operatorname{Spec} A$ be an affine open subset of $\operatorname{Mult}^{n}(X)$. We want to describe an affine open cover for the inverse image of $U$ in $\operatorname{Mult}^{n+1}(X)$, denoted Mult $\left.{ }^{n+1}(X)\right|_{U}$. With notation as in remark 4.6, the family $W_{i}$ is defined over $U$ by the ideal $J_{i}=\Gamma\left(U \times X, \mathscr{J}_{i}\right)$ in the affine coordinate ring of $U \times X$. Then

$$
\left.\operatorname{Mult}^{n+1}(X)\right|_{U}=\mathrm{P}(M)
$$

where

$$
M=\Gamma\left(U, \mathscr{F}_{n}\right)=J_{n} / \sum_{v=0}^{n-1} J_{v+1} J_{n-v}
$$

considered as an $A$-module. To give concrete equations for $\mathrm{P}(M)$, choose a free presentation

$$
A^{r} \stackrel{\left(g_{i j}\right)}{\longrightarrow} A^{s} \stackrel{\left(f_{j}\right)}{\longrightarrow} M \longrightarrow 0 .
$$

Then $\mathrm{P}(M)=\operatorname{Proj} R$ where

$$
R=A\left[t_{1}, \ldots, t_{s}\right] /\left(\sum_{j} g_{1 j} t_{j}, \ldots, \sum_{j} g_{r j} t_{j}\right) .
$$

Thus $\mathrm{P}(M)$ is covered by the affine open subsets $V_{i}=\operatorname{Spec} R_{i}$ where $R_{i}$ is the degree 0 part of the localization $R_{t_{i}}$. The universal quotient is the homomorphism

$$
M \otimes R_{i} \longrightarrow R_{i} \longrightarrow 0
$$

sending $f_{j} \otimes 1$ to $T_{j}=t_{j} / t_{i}$ (in particular $f_{i} \otimes 1 \mapsto 1$ ). Hence, on $V_{i}$ the universal flag is defined by ideals

$$
\widetilde{J}_{1} \supset \cdots \supset \widetilde{J}_{n+1}
$$

where $\widetilde{J}_{v}=J_{v} R_{i}$ for $v \leq n$, and

$$
\widetilde{J}_{n+1}=\left(T_{j} f_{i}-f_{j}\right)_{j \neq i}+\left(\sum_{v=0}^{n-1} J_{v+1} J_{n-v}\right) R_{i}
$$

As long as the rings $R_{i}$ are nilpotent-free, this gives an algorithm for computing an open cover of $\operatorname{HMF}(n)$. Otherwise we should divide by the nilradical to get the underlying reduced scheme. It turns out that in all our examples, i.e. whenever $n \leq 7$, $\operatorname{Mult}^{n}(X)$ is already reduced, hence $\operatorname{HMF}(n)=$ $\operatorname{Mult}^{n}(X)$. We do not know whether this is true for arbitrary $n$. 
Clearly, $\operatorname{Mult}^{2}(X)=H M F(2) \cong H(2) \cong \mathrm{P}^{1}$. The next result describes $H M F(3)$ and $H M F(4)$. We are going to use the following (well known and easy to derive) classification of punctual subschemes of length 2 and 3 on a nonsingular surface: For a suitable choice of local parameters, any subscheme of length two may be defined by an ideal of the form

$$
\left(x, y^{2}\right) \subset \mathscr{O}_{S, p} .
$$

Thus any such subscheme is curvilinear. For subschemes of length three, there are two types: Firstly there are the curvilinear ones, which for a suitable choice of local parameters may be defined by an ideal of the form

$$
\left(x, y^{3}\right) \subset \mathscr{O}_{S, p} .
$$

Secondly there is just one non curvilinear subscheme of length three, namely the first infinitesimal neighbourhood of $p$, defined by

$$
\mathfrak{m}_{p}^{2}=\left(x^{2}, x y, y^{2}\right) \subset \mathscr{O}_{S, p} .
$$

THEOREM 6.1. For $n=2$ and 3 the sheaf $\mathscr{F}_{n}$ is locally free of rank 2, hence $\operatorname{HMF}(n+1)$ is a $\mathrm{P}^{1}$-bundle over $\operatorname{HMF}(n)$. In particular, $H M F(3)$ and $H M F(4)$ are nonsingular.

Proof. Any point in $H M F(2)$ is curvilinear, hence $\mathscr{F}_{2}$ has rank two everywhere. Thus it is locally free.

A punctual subscheme of length 3 is either the first order infinitesimal neighbourhood of $p$ or it is curvilinear. Consider a point in $H M F(3)$, that is a filtration of ideals

$$
I_{3} \subset I_{2} \subset I_{1}=\mathfrak{m}_{p} .
$$

If $I_{3}$ is curvilinear, then

$$
I_{3} /\left(I_{1} I_{3}+I_{2}^{2}\right)=I_{3} / I_{1} I_{3}
$$

is two dimensional as before. If not, then $I_{3}=\left(x^{2}, x y, y^{2}\right)$. For a suitable choice of local parameters we may assume $I_{2}=\left(x, y^{2}\right)$. Then

$$
I_{1} I_{3}+I_{2}^{2}=\left(x^{2}, y^{3}, x y^{2}\right)
$$

and hence

$$
I_{3} /\left(I_{1} I_{3}+I_{2}^{2}\right)=\left\langle x y, y^{2}\right\rangle
$$

is two dimensional. Thus $\mathscr{F}_{3}$ has rank two everywhere. 
The surface $H M F(3)$ can be determined completely. In fact it is isomorphic to the minimal ruled surface $\mathrm{F}_{3}$. For this, let $R=k\left[a_{0}, a_{1}\right]$, then $\operatorname{HMF}(2)=$ $H(2)=\operatorname{Proj} R$ with universal family defined by the ideal

$$
J=\left(a_{1} y-a_{0} x, x^{2}, x y, y^{2}\right) \subset R \otimes_{k} \mathcal{O}_{X, p} .
$$

Then the sheaf $\mathscr{F}_{2}$ corresponds to the graded $R$-module $N$ with generators

$$
\begin{array}{lll}
f=a_{1} y-a_{0} x & g=x^{2} \\
h=x y & k=y^{2}
\end{array}
$$

where $f$ has degree 1 and the rest have degree 0 . The relations are

$$
a_{1} h=a_{0} g \quad a_{1} k=a_{0} h .
$$

From this we conclude that $N$ is isomorphic to $R(-1) \oplus R(2)$ in positive degrees, where $f$ generates the summand corresponding to $R(-1)$, and $g, h$ and $k$ generate the summand corresponding to $R(2)$. Thus

$$
\mathscr{F}_{2}=\mathscr{O}_{\mathrm{Pl}^{1}}(-1) \oplus \mathcal{O}_{\mathrm{P}^{1}}(2)
$$

and the associated projective bundle is $\mathrm{F}_{3}$.

Finally, we remark that $H F(4)$ is reducible, so $H M F(4)=H F^{\prime}(4)$ is not the only component. In fact, above the rational curve in $H F(3)=H M F(3)$ consisting of filtrations of the form

$$
\mathrm{m}_{p}^{2}=I_{3} \subset I_{2} \subset I_{1}=\mathfrak{m}_{p}
$$

where $I_{2}$ varies freely in a $\mathrm{P}^{1}$, every fibre in $H F(4)$ is a $\mathrm{P}^{2}$. Thus the inverse image of this curve has dimension 3 , which therefore cannot be contained in the irreducible three dimensional variety $H M F(4)$. To give an explicit example, the ideals

$$
\left(x^{2}, x y, y^{3}\right) \subset\left(x^{2}, x y, y^{2}\right) \subset\left(x^{2}, y\right) \subset(x, y)
$$

define a point in $H F(4)$ which is not multiplicative.

For $n=5$ we obtain the following, which corrects [9, Theorem 1].

THeOREM 6.2. $\operatorname{HMF}(5)$ is singular along a curve, but irreducible.

Proof. We compute the restriction of $H M F(5)$ to a particular open affine chart $U_{4} \subset H M F(4)$. By the same method one can compute an open cover explicitly.

With notation as in equation (16), let $U_{2} \subset H M F(2)$ be the open affine subset defined by $a_{0} \neq 0$. Then

$$
U_{2}=\operatorname{Spec} k[a]
$$


where $a=a_{1} / a_{0}$, and the universal flag is defined by the ideals

$$
J_{1}=(x, y) \quad J_{2}=\left(a y-x, y^{2}\right) .
$$

Carrying through the recipe given above, we find

$$
\left.H M F(3)\right|_{U_{2}}=\operatorname{Proj} k[a]\left[b_{0}, b_{1}\right]
$$

where the generators $b_{i}$ correspond to $t_{i}$ in equation (15). We define the open affine $U_{3} \subset H M F(3)$ by $b_{0} \neq 0$, then the universal flag on $U_{3}$ is defined by ideals $J_{1} \supset J_{2} \supset J_{3}$, where $J_{1}$ and $J_{2}$ are the ideals in (17) and

$$
J_{3}=\left(b(a y-x)-y^{2},(a y-x) x,(a y-x) y\right)
$$

where $b=b_{1} / b_{0}$. (We should really write $J_{1} k[a, b]$ and $J_{2} k[a, b]$ in place of $J_{1}$ and $J_{2}$, but this shouldn't cause any confusion.) Since

$$
a((a y-x) y)-(a y-x) x=(a y-x)^{2} \in J_{2}^{2}
$$

we find that $U_{3}$ trivializes $\mathscr{F}_{3}$ and

$$
\left.H M F(4)\right|_{U_{3}}=\operatorname{Proj} k[a, b]\left[c_{0}, c_{1}\right] .
$$

where again the new coordinates $c_{i}$ correspond to $t_{i}$ in equation (15). Define $U_{4} \subset H M F(4)$ by $c_{0} \neq 0$, then the universal flag is defined over $U_{4}$ by

$$
J_{4}=\left(c\left(b(a y-x)-y^{2}\right)-(a y-x) y, b(a y-x) y-y^{3},(a y-x)^{2}\right)
$$

where $c=c_{1} / c_{0}$, together with $J_{1}, J_{2}, J_{3}$ as above.

Now we are in position to describe the restriction of $H M F(5)$ to $U_{4}$. The module

$$
M=J_{4} /\left(J_{1} J_{4}+J_{2} J_{3}\right)
$$

is generated by

$$
\begin{aligned}
& f=c\left(b(a y-x)-y^{2}\right)-(a y-x) y \\
& g=b(a y-x) y-y^{3} \\
& h=(a y-x)^{2}
\end{aligned}
$$

and the element $b h-c f$ is contained in $J_{2} J_{3}$, thus

$$
\left.H M F(5)\right|_{U_{4}}=\operatorname{Proj} k[a, b, c][F, G, H] /(b H-c F) .
$$

In fact, since this is irreducible, reduced and of dimension four, the found relation $b h-c f$ is the only one. 
Thus $\left.H M F(5)\right|_{U_{4}}$ is irreducible and singular along a curve. Repeating the calculations while moving $U_{4}$ around proves the statement.

By the same procedure one may test the irreducibility of $\operatorname{HMF}(n)$, and hence question 5.5, for higher $n$. The explicit calculations get rather involved, but with the aid of the computer program Singular [3], using a primary decomposition algorithm, it has been verified that $\operatorname{HMF}(n)$ is irreducible for $n \leq 7$, and also that $\operatorname{Mult}^{n}(X)$ is already reduced. At 8 points we stopped due to lack of computer power.

\title{
REFERENCES
}

1. Briançon, Joël, Description de Hilb ${ }^{n} C\{x, y\}$, Invent. Math. 41 (1977), 45-89.

2. Ellingsrud, Geir, and Lehn, Manfred, Irreducibility of the punctual quotient scheme of a surface, Ark. Mat. 37 (1999), 245-254.

3. Greuel, G.-M., Pfister, G., and Schönemann, H., Singular 2.0, A Computer Algebra System for Polynomial Computations, Centre for Computer Algebra, University of Kaiserslautern, 2001, http://www.singular.uni-kl.de.

4. Grothendieck, Alexander, Éléments de géométrie algébrique. III. Étude cohomologique des faisceaux cohérents. I, Inst. Hautes Études Sci. Publ. Math. (1961), 167.

5. Grothendieck, Alexander, Techniques de construction et théorèmes d'existence en géométrie algébrique. IV. Les schémas de Hilbert, Séminaire Bourbaki, Vol. 6, Soc. Math. France, Paris, 1995, pp. Exp. No. 221, 249-276.

6. Iarrobino, Anthony A., Punctual Hilbert schemes, Mem. Amer. Math. Soc. 10 (1977), no. 188, viii+112.

7. Le Barz, Patrick, Platitude et nonplatitude de certains sous-schémas de $\operatorname{Hilb}^{k} \mathbf{P}^{N}$, J. Reine Angew. Math. 348 (1984), 116-134.

8. Tikhomirov, Alexander S., A smooth model of punctual Hilbert schemes of a surface, Trudy Mat. Inst. Steklov. 208 (1995), 318-334.

9. Tikhomirov, Sergei A., Punctual Hilbert schemes of small length in dimensions 2 and 3, Mat. Zametki 67 (2000), no. 3, 414-432.

\author{
DEPARTMENT OF MATHEMATICS \\ UNIVERSITY OF OSLO \\ P.O. BOX 1053 \\ $\mathrm{N}-0316$ OSLO \\ NORWAY \\ E-mail: martingu@math.uio.no
}

